- M. J. DE JoAnnis signale la capture faite par M. L. RADot, à l'Escoriai le 10 mai 1912, d'un exemplaire ơ très frais de Christophia ruscinonella Rag. [Lep. Phycitinae]. Ce bel exemplaire concorde absolument avec l'exemplaire type, provenant de Perpignan, qui se trouve dans la collection Ragonot. Cette espèce paraît jusqu'ici fort rare et n'avait pas été signalée, semble-t-il, depuis la capture du type; elle a peut-être son centre dans la péninsule ibérique et l'exemplaire de Perpignan proviendrait des confins de son habitat. A l'avenir de le dire.

Rectification synonymique [LEP.]. - M. A. JANET présente la note synonymique suivante :

La première figure de la planche 94 du volume IX de Seitz (faune Indo-Australienne, Diurnes) représente sous le nom d'Anadebis batmara une forme de Satyride provenant de Chiem-Hoa (Tonkin central), traitée dans le texte (p. 328 de l'édition française) comme forme d'A. diademoides Moore.

A. batmara a été décrit par FrüHstorfer dans Iris, vol. XX [1907], p. 253.

Or cette forme n'est autre que celle que j'ai décrite en 1896 dans le Bulletin de la Société entomologique de France (p. 216) sous le nom de Zethera Noirei.

A l'appui de la note synonymique ci-dessus, M. A. Janet fait passer sous les yeux des membres de la Société la planche en question de l'ouvrage de SeItz et le type de Zethera Noirei, qui provient de Son-tay (Tonkin), ainsi qu'un co-type, de Caï-vong (Tonkin).

\title{
Gommunications.
}

\section{Description de deux Cetonides nouveaux [Col. Scarabaeidae]}

\author{
par A. Bourgoin.
}

Pachnoda Alluaudi, n. sp. - Capite dense punctato, clypeo leviter emarginato, ante apicem transverse impresso, in medio convexo; pronoto flavo-testaceo minutissime et densissime punctato, hoc maculis duabus oblongis discoidalibus nigris; elytris sat regulariter punctatis; flavo-testaceis, humeris nigro-punctatis; tuberculis subapicalibus nigris. Corpus subtus nigro-viride; coxis posticis ad latera pedibusque brunneorufis. 
Voisin de $\boldsymbol{P}$. inscripta G. et P.; taille et coloration presque semblables. En diffère par les taches noires du pronotum formant sur le disque denx bandes longitudinales obliquement tronquées intérieurement en arrière; par les élytres plus finement ponctués, ayant seulement un point huméral et le calus apical noirs; par le métasternum et l'abdomen sans taches blanches sur les côtés, et surtout par la saillie mésosternale plane, mince, presque circulaire, trois fois aussi large en son milieu qu'à la suture méso-métasternale.

Longueur du bord antérieur du pronotum à l'extrémité des élytres : 22 à 25 mill.

Largeur aux épaules : 12,ä à 14 mill.

Afrique orientale anglaise (Kikuyu Escarpment) : Kijabé, alt. $2.100 \mathrm{~m}$.

Voyage de Ch. Alluaud et R. Jeannel.

$\sigma^{\top}$ et $\bigcirc$. Types : Coll. du Muséum de Paris et coll. A. Bourgorn.

Pygora sanguineomarginata, n. sp. - Supra et infra nitida; caput violaceum, sat regulariter punctatum; clypeo in medio elevato, antico leviter sinuato. Pronoto violaceo, subtilissime punctato; scutello viridi; elytra quadristriata, antice violacea, postice viridia, sanguineomarginata.

Subtus viridis, ad latera albo-maculata; femora cyaneo viridia, tibiis violaceis, tarsis violaceo-viridibus.

Pygidium convexum viride, sparsissime punctatum.

$\sigma^{x}$ Tête indigo, sauf la partie médiane et les bords du clypéus qui sont verts; scutellum vert sombre, assez aigu. Pronotum indigo, unicolore, à ponctuation extrêmement fine, à rebord non élargi au milieu et ne formant pas d'angle sensible.

Élytres indigo, passant au vert en arrière, à bordure marginale rouge-sang visible sous toutes les incidences.

Prosternum et saillie mésosternale bleus; le reste du dessous vert, maculé de blanc sur les còtés du pectus et de l'abdomen; segments abdominaux 1-4 légèrement concaves et maculés de blanc en leur milieu; fémurs verts à la base, violacés à leur sommet; tibias violacé rougeâtre, tarses bleu violet foncé.

Longueur, du bord antérieur du pronotum à l'extrémité des élytres : 12 mill.

Largeur aux épaules : 6,כ̈ mill.

$1 \sigma^{\top}$. Madagascar : Mahatsinjo. Type : coll. A. Bourgorn. 


\section{$2 \mathrm{BHL}$ Biodiversity Heritage Library}

1913. "Description de deux Cetonides nouveaux." Bulletin de la Société entomologique de France 1913, 290-291.

https://doi.org/10.5962/bhl.part.16291.

View This Item Online: https://www.biodiversitylibrary.org/item/36377

DOI: https://doi.org/10.5962/bhl.part.16291

Permalink: https://www.biodiversitylibrary.org/partpdf/16291

\section{Holding Institution}

Smithsonian Libraries

\section{Sponsored by}

Smithsonian

\section{Copyright \& Reuse}

Copyright Status: NOT_IN_COPYRIGHT

This document was created from content at the Biodiversity Heritage Library, the world's largest open access digital library for biodiversity literature and archives. Visit BHL at https://www.biodiversitylibrary.org. 